\title{
Muscle Force-Velocity Relationships Observed in Four Different Functional Tests
}

\author{
by \\ Milena Z. Zivkovic ${ }^{1}$, Sasa Djuric ${ }^{1}$, Ivan Cuk ${ }^{1,2}$, Dejan Suzovic ${ }^{1}$, Slobodan Jaric ${ }^{3,4}$
}

The aims of the present study were to investigate the shape and strength of the force-velocity relationships observed in different functional movement tests and explore the parameters depicting force, velocity and power producing capacities of the tested muscles. Twelve subjects were tested on maximum performance in vertical jumps, cycling, bench press throws, and bench pulls performed against different loads. Thereafter, both the averaged and maximum force and velocity variables recorded from individual trials were used for force-velocity relationship modeling. The observed individual force-velocity relationships were exceptionally strong (median correlation coefficients ranged from $r=0.930$ to $r=0.995$ ) and approximately linear independently of the test and variable type. Most of the relationship parameters observed from the averaged and maximum force and velocity variable types were strongly related in all tests $(r=0.789-0.991)$, except for those in vertical jumps $(r=0.485-0.930)$. However, the generalizability of the force-velocity relationship parameters depicting maximum force, velocity and power of the tested muscles across different tests was inconsistent and on average moderate. We concluded that the linear force-velocity relationship model based on either maximum or averaged force-velocity data could provide the outcomes depicting force, velocity and power generating capacity of the tested muscles, although such outcomes can only be partially generalized across different muscles.

Key words: power; parameter; output; load; generalizability.

\section{Introduction}

The force-velocity (F-V) relationship of the muscles performing multi-joint maximum performance tasks has been the focus of recent research. An important reason for that was the possibility to selectively assess force $(\mathrm{F})$, velocity $(\mathrm{V})$ and power $(\mathrm{P})$ generating capacity of muscles performing functional movements that cannot be assessed from a single movement condition typically applied in routine testing procedures. Moreover, the F-V relationship could be of an approximately linear shape instead of a hyperbolic one that is typically observed from either in vitro muscles or individual muscle groups (Jaric, 2015). The linear shape of the F-V

relationship not only simplifies its assessment from various functional movement tasks, but the relationship parameters also directly reveal the capacities of the tested muscles to develop high $\mathrm{F}$, $\mathrm{V}$ and $\mathrm{P}$ output. Namely, a range of $\mathrm{F}$ and $\mathrm{V}$ data observed from different loading conditions has been modeled by a linear regression model:

$$
\mathrm{F}(\mathrm{V})=\mathrm{F}_{0}-\mathrm{aV}
$$

where parameter $\mathrm{a}$ is the relationship slope. $F_{0}$ (i.e., F-intercept) corresponds to the maximum force of the tested muscles that enables calculation of the $\mathrm{V}$-intercept:

\footnotetext{
1 - University of Belgrade - Faculty of Sport and Physical Education, The Research Centre, Belgrade, Serbia.

2 - College of Sports and Health, Belgrade, Serbia.

3 - University of Delaware, Department of Kinesiology and Applied Physiology, Newark, USA.

4 - University of Delaware, Biomechanics and Movement Science Graduate Program, Newark, USA.
} 


$$
\mathrm{V}_{0}=\mathrm{F}_{0} / \mathrm{a}
$$

revealing the maximum velocity of the tested muscles. Finally, maximum power of the tested muscles is:

$$
\mathrm{P}_{0}=\mathrm{F}_{0} \mathrm{~V}_{0} / 4
$$

Relatively strong and approximately linear F-V relationships have been observed in various maximum performance functional tests (e.g., cycling, squats and vertical jumps, leg extensions performed against various sledge devices and dynamometers (Hahn et al., 2014; Samozino et al., 2012, 2014; Yamauchi et al., 2009), running or arms and upper body movements), while the relationship parameters also proved to be highly reliable and at least moderately valid (Jaric, 2015). However, some other findings showed weaker and somewhat non-linear F-V relationships (Allison et al., 2013; Feeney et al., 2016; Hahn et al., 2014; Limonta and Sacchi, 2010). Therefore, direct comparisons of F-V relationships observed in different tests could reveal whether those differences originate either from inherent differences among various tests and the involved muscles, or from somewhat different methodologies applied in different studies.

Regarding other unresolved issues related to the F-V relationships obtained from functional multi-joint tests, note that there is no general recommendation yet regarding whether the averaged or maximum $\mathrm{F}$ and $\mathrm{V}$ variable types should be used for modeling of the muscular F-V relationships (Jaric, 2015). The relationships observed from the maximum $\mathrm{F}$ and $\mathrm{V}$ variable types expectedly reveal higher $F_{0}$ and $V_{0}$, and therefore higher $\mathrm{P}_{0}$ than the relationships observed in the averaged $\mathrm{F}$ and $\mathrm{V}$ variable types (Cuk et al., 2014; Sreckovic et al., 2015). However, particular advantages of either type of the $\mathrm{F}$ and $\mathrm{V}$ variables have not been explored yet. Finally, it should be kept in mind that the implicit presumption of any routine battery of the tests of muscle function is that the outcomes typically observed in very few tests and muscles can be partly generalized to other muscular systems performing different functional movements (Bohannon, 2008; Pojednic et al., 2012). However, the relationships among the muscle capacities assessed through $\mathrm{F}_{0}, \mathrm{~V}_{0}$ and $\mathrm{P}_{0}$, obtained from different tests have not been assessed yet.

To address the discussed problems we designed a study with the purpose to both explore and compare the F-V relationships observed both in different functional tests, as well as in different types of $\mathrm{F}$ and $\mathrm{V}$ variables. Our first hypothesis was that the explored F-V relationships would be strong and approximately linear. The second hypothesis was that the F-V relationship parameters $F_{0}, V_{0}$ and $P_{0}$ would be higher when obtained from maximum than when obtained from the averaged $\mathrm{F}$ and $\mathrm{V}$ variables. The third hypothesis was that the muscle capacities obtained through the F-V relationship parameters would be generalizable across different functional tests. The results were expected to expand our knowledge regarding the mechanical capacities and function of our muscular system, as well as to contribute to further development of routine tests of muscle F-, V- and P-generating capacity applied in various human movement related areas.

\section{Material and Methods}

\section{Participants}

The sample size estimate based on the Cohen guidelines (Cohen, 1988) was conducted for an alpha level of 0.05 and power of 0.8 using our previous studies (Cuk et al., 2014; Sreckovic et al., 2015). The sample sizes ranging from 3 to 12 appeared to be necessary to detect differences among dependent variables obtained from different loading conditions. Therefore, we recruited 12 healthy male subjects (age $22.1 \pm 3.4$ yrs; body height $184.1 \pm 7.1 \mathrm{~cm}$; body mass $80.8 \pm$ $8.2 \mathrm{~kg}$; data shown as mean $\pm \mathrm{SD}$ ). Their body mass index was $24.5 \pm 1.5 \mathrm{~kg} / \mathrm{m}^{2}$, and the body fat percentage $11.2 \pm 2.8 \%$. Their physical activity level was assessed via the IPAQ questionnaire (Taylor-Piliae et al., 2006), in which 4 subjects revealed high, 5 moderate and the remaining 3 revealed a low level of physical activity. None of them were an active athlete, and none reported medical problems or recent injuries. They were informed regarding the potential risks associated with the applied testing protocol. They were also instructed to avoid any unusual strenuous activities over the course of the study. All subjects gave written informed consent to the experiment, which was in accordance with the Declaration of Helsinki and approved by the Institutional 
Review Board of the Faculty of Sport and Physical Education, University of Belgrade.

\section{Procedures}

In addition to body height and body mass, the body fat percentage was also calculated (a bioelectric impedance method; In Body 720; USA). The main part of the experimental procedure consisted of the following 4 maximum performance functional tests conducted under different loads: countermovement jumps (CMJ), a short anaerobic Wingate test (CYCLING), bench press throws (B-PRESS), and bench pulls (BPULL) (Figure 1).

CMJ were performed on a force plate (AMTI, BP600400; USA), where subjects wore a weighted west and belt (MiR Vest Inc; USA). Subjects were instructed to perform unconstrained maximum vertical jumps from a standing upright position with their arms akimbo. The rest period between consecutive jumps was 1 min and 2-3 $\mathrm{min}$ between different loading magnitude (Markovic et al., 2013).

CYCLING consisted entirely of testing the maximum power output throughout the $6 \mathrm{~s}$ maximal cycling sprint (Logan et al., 2000; Mendez-Villanueva et al., 2007; Pazin et al., 2011) performed on a Monark 834E leg cycle ergometer (Monark, Varberg, Sweden). Subjects were instructed to perform an "all out" effort from the very beginning of the test (Pazin et al., 2011). They began the exercise with their preferred leg with the crank positioned at $45^{\circ}$ forward. Subjects were also instructed to remain seated during the entire sprint. The seat height was individually adjusted and toe clips with straps were used to prevent the feet from slipping off the pedals. During each trial a strong verbal encouragement was provided. The rest period between consecutive sprints was set to 4 min (Pazin et al., 2011).

B-PRESS were performed on a Smith machine (Sreckovic et al., 2015). Mechanical stops fixed the initial bar position at about $1 \mathrm{~cm}$ above the chest, while two stoppers were catching the bar during its descending trajectory. The subjects were instructed to throw the bar as high as possible. The rest periods between two consecutive bench press throws were $45 \mathrm{~s}$, while the rest between different loading conditions was about 3 min (Leontijevic et al., 2013; Sreckovic et al., 2015).

B-PULL were performed according to the already established protocol (Sanchez-Medina et al., 2013). Subjects were instructed to lay face down on the high bench while placing their chin on the padded edge of the bench. They pulled the bar upwards with maximum effort until the bar struck the underside of the bench. The lower part of their legs and their upper body were both strapped to the bench with padded rope. The rest periods were the same as in B-PRESS.

In total, each subject completed 4 sessions, separated by rest periods of 5-7 days (Cuk et al., 2014; Sreckovic et al., 2015). The first testing session consisted of anthropometric measurements, followed by familiarization with two functional tests. The second session consisted of familiarization with two additional tests. The third and fourth testing sessions were used for data collection. Note that the order of the tests was randomized for each subject. To avoid fatigue each familiarization and testing session involved only one test of upper limbs and one test of lower limbs. The sessions typically lasted about $90 \mathrm{~min}$. For all tests except CYCLING, the first trial was considered to be a practice trail, while the second trial was used for further analysis. According to both the subjects' reports and our previous studies, fatigue was never an issue. Standard 10 min stretching and warm up procedures were conducted prior to each test of lower (Cuk et al., 2014; Markovic et al., 2013) and upper limbs (Sanchez-Medina et al., 2006; Sreckovic et al., 2015).

To simplify both the testing procedure and presenting the data averaged across the subjects, the absolute loads (instead of the load magnitude relative to subjects' strength) were selected. The CMJ test always started with the load originating from the emptied vest and belt only (approximately $1 \mathrm{~kg}$ ), and then continued with the consecutive addition of 8, 16, 24 and 32 $\mathrm{kg}$ of weights. Namely, the familiarization procedure revealed that all subjects were able to jump with $32 \mathrm{~kg}$ of the external load. In total, the subjects performed 10 jumps (5 loads $\mathrm{x} 2$ trials). Regarding CYCLING, subjects performed 5 sprints with the external load starting with $2 \mathrm{~kg}$, followed by the addition of 2, 4, 6 and $8 \mathrm{~kg}$ of weights (i.e., from 2 to $10 \mathrm{~kg}$; 5 loads $\times 1$ trial). Since the individual maximum load lifted in BPRESS and B-PULL was within the range between 62 and $90 \mathrm{~kg}$, the applied external loads were 20, 
27.5, 35, 42.5, 50 and $57.5 \mathrm{~kg}$. Note that the minimum load of $20 \mathrm{~kg}$ consisted of the bar and arm segments (Sreckovic et al., 2015), while the weight plates were added for higher loads. In total, subjects completed 12 bench press throws, as well as 12 bench pulls (i.e., 6 loads $\times 2$ trials each).

Analysis

Regarding the CMJ, a custom-designed LabVIEW (National Instruments 2013; USA) program was used to acquire and process the vertical component of the ground reaction $\mathrm{F}$. The signals were sampled at $1000 \mathrm{~Hz}$ and low-pass filtered (a second-order recursive low-pass Butterworth filter with a cut-off frequency of 10 $\mathrm{Hz}$ ). Integration of the acceleration signal obtained from $\mathrm{F}$ was conducted to calculate $\mathrm{V}$ (Cuk et al., 2014). The analyzed movement phase covered the time interval from the lowest position of the body's center of mass to the initiation of the flight phase. Regarding CYCLING, Monark anaerobic test software was used to acquire $\mathrm{P}$ and the frequency data. To assess the corresponding linear measures, $\mathrm{V}$ was calculated from the frequency and crank length, while $F$ was calculated through the division of $\mathrm{P}$ by $\mathrm{V}$.

Another custom-made LabVIEW program was used to assess $\mathrm{F}$ and $\mathrm{V}$ from B-PRESS and BPULL. 3D kinematic recording (Qualisys $A B$, Gothenburg, Sweden) sampled at the rate of 240 $\mathrm{Hz}$ with a recursive Butterworth low-pass filter (cutoff frequency of $10 \mathrm{~Hz}$ ) was used to assess the vertical position of the bar. $\mathrm{V}$ and the acceleration of the bar were calculated from the first and second derivative of the position, respectively, while $\mathrm{F}$ was calculated from the weight and inertia of the total mass lifted. In line with previous studies (Sanchez-Medina et al., 2013; Sreckovic et al., 2015), the analyzed concentric phase covered the time interval from movement initiation to either the termination of the handsbar contact (in B-PRESS) or the impact of the bar with the bench (B-PULL). When the termination of the hand-bar contact was not recorded, the concentric phase termination was set to the instant when $\mathrm{V}$ dropped to $5 \%$ of the maximum V.

The average and maximum values of $\mathrm{F}$ and $\mathrm{V}$ variable types were separately calculated for all tests. In CYCLING specifically, the software separately calculated $\mathrm{P}$ and frequency for each consecutive second that allowed for the calculation of both the averaged and maximum $\mathrm{F}$ and $\mathrm{V}$ variable types.

Statistical analyses were conducted on the parameters of the F-V relationships obtained from 4 different tests separately, which were calculated from the averaged and maximum $\mathrm{F}$ and $\mathrm{V}$ variable types. Descriptive statistics were calculated as means, standard deviations and the coefficient of variation, while correlation coefficients were presented through their median values and ranges. Prior to the statistical analyses, initial testing revealed that none of the dependent variables significantly deviated from their normal distribution (Kolmogorov-Smirnov test). Both the linear and the $2^{\text {nd }}$ order polynomial regression models were used to assess the averaged values across the subject F-V relationships. The corresponding $95 \%$ confidence intervals ( $95 \% \mathrm{CI}$ ) were also calculated to compare the strength of the F-V relationships obtained from the linear and polynomial regression models applied to the averaged across the subject's data. A Student's ttest for dependent samples was used to evaluate the differences between the relationship parameters observed in the averaged and maximum $\mathrm{F}$ and $\mathrm{V}$ variable types. Pearson's correlations were also used to test the relationships between the same parameters obtained from the averaged and maximum $\mathrm{F}$ and $\mathrm{V}$ variable types, as well as among the same parameters obtained from 4 tests. Two-way mixed models ANOVA with Bonferroni post-hoc tests were applied to assess the differences among the Z-transformed correlation coefficients obtained from different tests and variable types. The level of statistical significance was set at $p<0.05$. All statistical analyses were performed using SPSS 21 (IBM, Armonk, NY).

\section{Results}

Figure 2 shows the linear and polynomial models of the F-V relationship obtained from four different tests. The individual data points represent averaged values across the subjects $F$ and $\mathrm{V}$ variable types for each individual load separately calculated from their averaged and maximum variable. Note that out of 8 polynomial models, 6 suggested a concave, while 2 suggested convex shape. Of particular importance could not only be that all regression models revealed 
exceptionally high correlation coefficients ( $\mathrm{r}>$ 0.980 ), but also that none of the coefficients of linear regression models were below the $95 \%$ CI of the correlation coefficients obtained from the corresponding polynomial regression models.

Table 1 shows the outcomes of the linear model applied to the individual data sets. As expected, the magnitude of the relationship parameters was higher when calculated from the maximum compared to the averaged $\mathrm{F}$ and $\mathrm{V}$ variable types, with the exceptions of $\mathrm{F}_{0}$ obtained from CYCLING. Steepness of the regression slopes suggests that significantly higher $\mathrm{P}_{0}$ observed from maximum $\mathrm{F}$ and $\mathrm{V}$ variable types in all 4 tests originates more from the differences in $\mathrm{V}_{0}$ than from the differences in $\mathrm{F}_{0}$. Note also that the visual inspection of the data suggests that the across subject variability of $\mathrm{F}_{0}$ and $\mathrm{V}_{0}$ calculated through coefficients of variation was significantly higher in CMJ than in the remaining 3 tests. Finally, the individual correlation coefficients of the applied linear model were exceptionally strong for all four tests and both types of variables. The only exception was CMJ, where some individual correlation coefficients were somewhat lower and approaching 0.80, while in the 3 remaining tests very few were below 0.95 . When the Z-transformed coefficients of correlation were subjected to 2-way mixed model ANOVA, the main effect of the test was significant $(\mathrm{F}=22.8 ; p<0.01)$, but not the main effect of the variable type $(\mathrm{F}=2.1 ; p=0.16)$ and their interaction $(\mathrm{F}=0.9 ; p=0.46)$. Specifically, the individual $\mathrm{F}-\mathrm{V}$ relationships were strongest for CYCLING (all $p<0.01$ ) and weakest for CMJ (all $p$ $<0.01$ ), while there were no differences between B-PRESS and B-PULL ( $p=0.99)$.

Figure 3 depicts the relationships between the same parameters of the individual linear F-V regression models observed in the maximum and averaged $\mathrm{F}$ and $\mathrm{V}$ variable types obtained from individual tests. The relationships appeared to be mainly strong and significant for all parameters except for Po observed in CMJ.

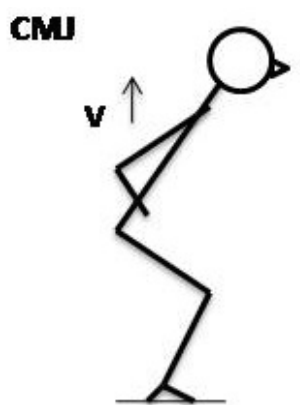

\section{CYCUNG}

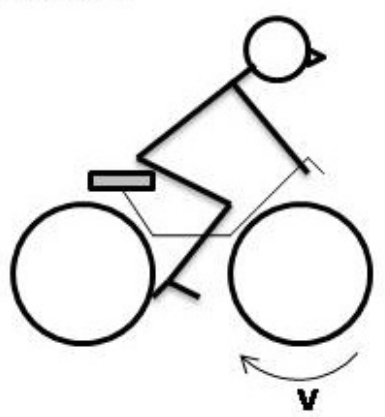

B-PUL

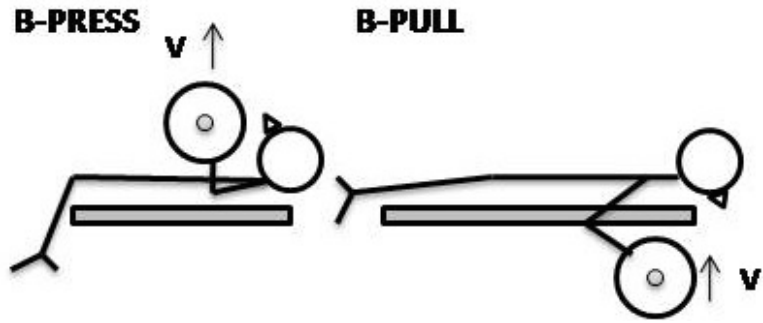

Figure 1

Illustration of the functional tests of leg (upper panels) and arm muscles (bottom panels). Specifically, CMJ, CYCLING, B-PRESS, B-PULL were conducted against variable external loads to provide a range of the averaged and maximum $F$ and $V$ data for further modeling 

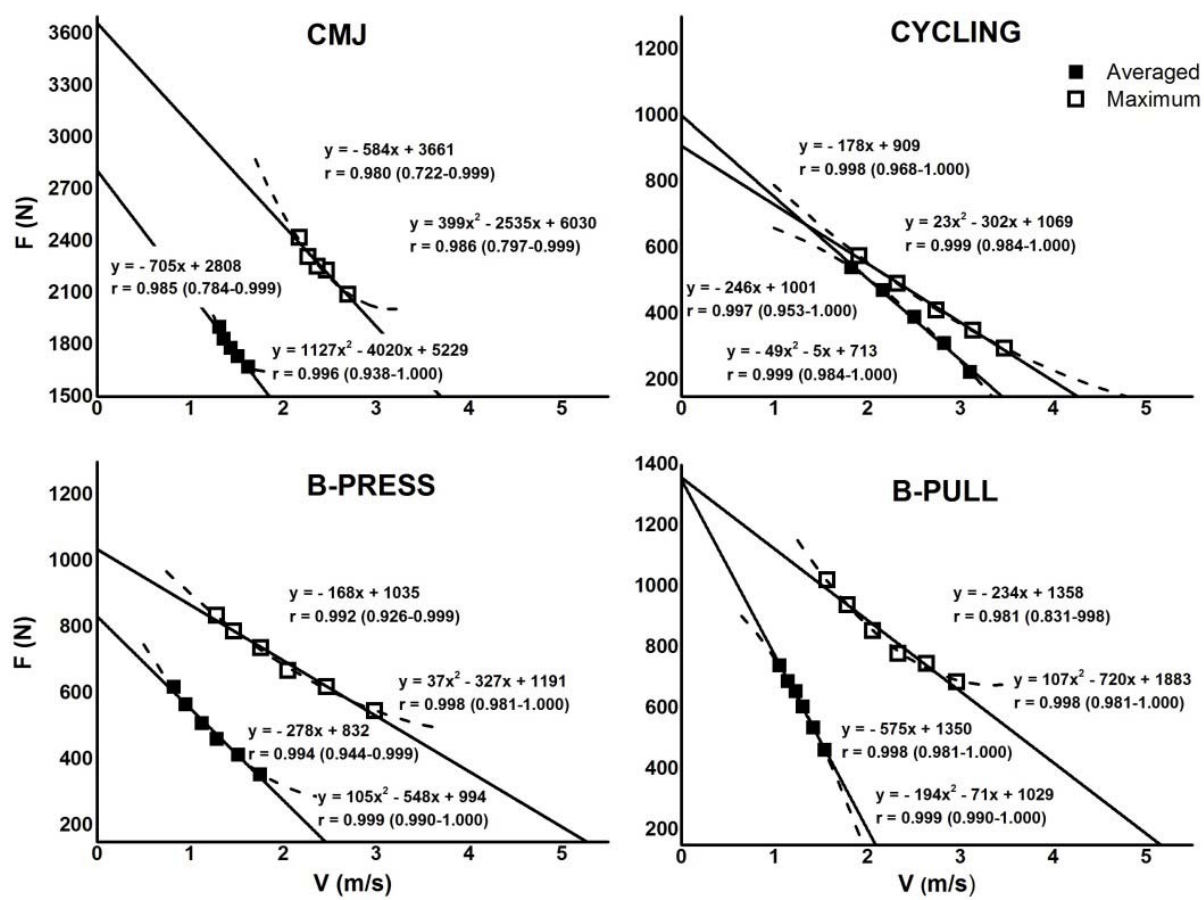

Figure 2

The linear (solid line) and second-order polynomial regression models (dashed line) applied to the averaged across the subject $F$ and $V$ variable types obtained from 4 different tests and shown separately for the averaged (filled squares) and maximum (open squares) $F$ and $V$ variable types.

The regression equations are shown with the corresponding correlation coefficients and $95 \%$ CI.

Table 1

Outcomes of individual F-V relationships obtained from 4 functional tests and 2 types of variables

\begin{tabular}{lcccccccc}
\hline \multirow{2}{*}{ Test } & Variable & $\mathrm{F}_{0}(\mathrm{~N})$ & $\begin{array}{c}\mathrm{CV} \mathrm{F} \\
(\%)\end{array}$ & $\mathrm{V}_{0}(\mathrm{~m} / \mathrm{s})$ & $\begin{array}{c}\mathrm{CV} \mathrm{V} \\
(\%)\end{array}$ & $\mathrm{P}_{0}(\mathrm{~W})$ & $\begin{array}{c}\text { CV } \mathrm{P}_{0} \\
(\%)\end{array}$ & $\mathrm{r}$ \\
\hline \multirow{2}{*}{ CMJ } & Averaged & $2948 \pm 788$ & 26.7 & $4.5 \pm 1.8$ & 38.9 & $3089 \pm 584$ & 18.9 & $0.951(0.877-0.992)$ \\
& Maximum & $3856 \pm 1177^{* *}$ & 30.5 & $6.9 \pm 2.0^{* *}$ & 28.7 & $6213 \pm 1003^{* *}$ & 16.1 & $0.930(0.815-0.996)$ \\
\multirow{2}{*}{ CYCLING } & Averaged & $1033 \pm 222$ & 21.5 & $4.1 \pm 0.3$ & 7.1 & $1047 \pm 189$ & 18.1 & $0.995(0.978-0.999)$ \\
& Maximum & $928 \pm 153^{* *}$ & 16.5 & $5.1 \pm 0.4^{* *}$ & 8.6 & $1181 \pm 171^{* *}$ & 14.5 & $0.992(0.980-0.999)$ \\
\multirow{2}{*}{ B-PRESS } & Averaged & $830 \pm 58$ & 7.0 & $3.0 \pm 0.3$ & 9.5 & $627 \pm 73$ & 11.7 & $0.984(0.963-0.991)$ \\
& Maximum & $1035 \pm 78^{* *}$ & 7.5 & $6.2 \pm 0.8^{* *}$ & 12.2 & $1610 \pm 201^{* *}$ & 12.5 & $0.983(0.952-0.997)$ \\
\multirow{2}{*}{ B-PULL } & Averaged & $1350 \pm 234$ & 17.3 & $2.4 \pm 0.3$ & 10.5 & $807 \pm 124$ & 15.3 & $0.990(0.940-0.997)$ \\
& Maximum & $1368 \pm 177$ & 12.9 & $5.9 \pm 0.8^{* *}$ & 14.3 & $2007 \pm 338^{* *}$ & 16.9 & $0.968(0.943-0.997)$ \\
\hline
\end{tabular}

CMJ, countermovement jump test; CYCLING, short Wingate anaerobic test;

$B$-PRESS, bench press throw test; B-PULL, bench pull test; Fo, force intercept;

$V_{0}$, velocity intercept, $P_{0}$, maximum power; $r$, correlation coefficients (medians and ranges); averaged, data observed from averaged $F$ and $V$ variables; maximum, data observed in maximum

F and $V$ variables;

$\left({ }^{*} p<0.05 ;{ }^{* *} p<0.01\right.$ - differences observed in averaged and maximum data) 

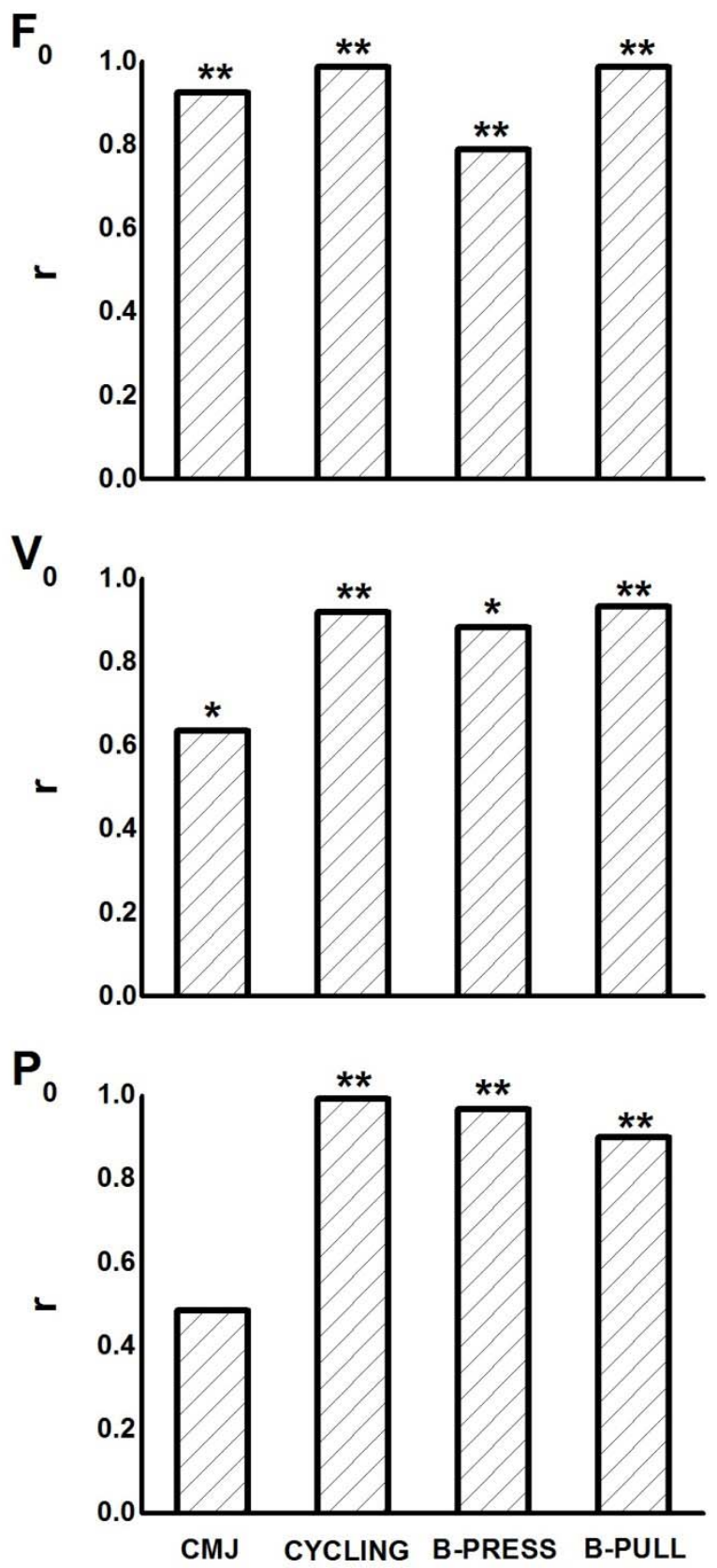

Figure 3

Correlation coefficients between the same $F-V$ regression parameters observed in the averaged and maximum $F$ and $V$ variable types in each

$$
\text { of } 4 \text { tests }\left({ }^{*} p<0.05 ;{ }^{* *} p<0.01\right)
$$


Table 2

Pearson's correlation coefficients observed among the same F-V parameters of 4 different tests obtained separately for the averaged and maximum $F$ and $V$ variables

\begin{tabular}{|c|c|c|c|c|c|c|c|c|c|}
\hline \multirow[b]{2}{*}{ Parameter } & \multirow[b]{2}{*}{ Test } & \multicolumn{4}{|c|}{ Averaged } & \multicolumn{4}{|c|}{ Maximum } \\
\hline & & $\mathrm{CMJ}$ & CYCLING & B-PRESS & B-PULL & CMJ & CYCLING & B-PRESS & B-PULL \\
\hline \multirow{4}{*}{$F_{0}$} & $\mathrm{CMJ}$ & 1 & & & & 1 & & & \\
\hline & CYCLING & 0.39 & 1 & & & $0.61^{*}$ & 1 & & \\
\hline & B-PRESS & -0.24 & 0.38 & 1 & & 0.20 & 0.49 & 1 & \\
\hline & B-PULL & 0.30 & 0.44 & 0.46 & 1 & 0.54 & 0.49 & $0.74^{* *}$ & 1 \\
\hline \multirow{4}{*}{$\mathrm{V}_{0}$} & $\mathrm{CMJ}$ & 1 & & & & 1 & & & \\
\hline & CYCLING & 0.25 & 1 & & & 0.27 & 1 & & \\
\hline & B-PRESS & 0.13 & 0.08 & 1 & & 0.20 & 0.30 & 1 & \\
\hline & B-PULL & -0.27 & -0.16 & 0.29 & 1 & -0.01 & 0.04 & 0.41 & 1 \\
\hline \multirow{4}{*}{$\mathrm{P}_{0}$} & $\mathrm{CMJ}$ & 1 & & & & 1 & & & \\
\hline & CYCLING & $0.64^{*}$ & 1 & & & 0.57 & 1 & & \\
\hline & B-PRESS & $0.67^{*}$ & $0.66^{*}$ & 1 & & 0.50 & $0.58^{*}$ & 1 & \\
\hline & B-PULL & 0.51 & $0.77^{* *}$ & 0.46 & 1 & 0.50 & $0.82^{* *}$ & 0.49 & 1 \\
\hline
\end{tabular}

Table 2 depicts the generalizability of the relationship parameters through the correlations among the same parameters obtained from 4 different tests. In general, the correlation coefficients ranged from weak to strong. A 2-way mixed model ANOVA was conducted on their Ztransformed values with the main factors being parameter and variable type. The results revealed the main effects of both the parameter $(\mathrm{F}=10.7 ; \mathrm{p}$ $<0.01)$ and variable type ( $\mathrm{F}=16.4 ; \mathrm{p}<0.01)$, as well as their interaction $(\mathrm{F}=9.9 ; \mathrm{p}<0.01)$. Specifically, the relationships were stronger among $P_{0}$ than among $\mathrm{V}_{0}$ parameters $(\mathrm{p}<0.01)$, as well as the parameters observed in the maximum versus those observed in the averaged $\mathrm{F}$ and $\mathrm{V}$ variable types.

\section{Discussion}

To our knowledge, this is the first study that comprehensively compares the F-V relationships obtained from both a number of functional performance tests and two types of $\mathrm{F}$ and $\mathrm{V}$ variables. In line with our first hypothesis, the findings suggest exceptionally strong and approximately linear F-V relationships independently of the tests and variable types. Regarding our second hypothesis, the results observed in the maximum $\mathrm{F}$ and $\mathrm{V}$ variable types expectedly revealed higher $\mathrm{P}_{0}$ than the relationships observed in averaged $\mathrm{F}$ and $\mathrm{V}$ variable types. This difference mainly originated from higher $V_{0}$, rather than from higher $F_{0}$. Regarding our third hypothesis, we found that the muscle capacities as assessed through the F-V relationship parameters obtained from muscles performing one test could be only partially generalized to other tests and muscles.

Overall, the strength of individual F-V relationships proved not only to be exceptionally high, but also comparable (Cuk et al., 2014; Garcia-Ramos et al., 2016; Jaskolska et al., 1999; Yamauchi et al., 2009), if not stronger than the 
same relationships observed in previous studies (Allison et al., 2013; Feeney et al., 2016; Rabita et al., 2015). In line with our recent studies (Cuk et al., 2014; Sreckovic et al., 2015), none of the data sets revealed significant differences between the linear and parabolic fit. Therefore, based on the presented robust set of data one could generally conclude that F-V relationships could be strong and approximately linear when observed in muscles performing various multi-joint functional tests. This finding also suggests that the selection of absolute loads should not have had noticeable effects on the observed outcomes. Nevertheless, the results also suggest that while the relationship could be particularly strong when obtained from CYCLING, it could also be somewhat weaker in $\mathrm{CMJ}$ than in the remaining three tests. A possible explanation could be based on the differences between the tested tasks regarding the adaptation of the movement patterns to different loads. Namely, while B-PRESS, B-PULL and particularly CYCLING do not allow for a prominent adaptation due to a limited number of kinematic degrees of freedom, CMJ allows for it through both the adaptation of countermovement depth and co-variation of ankle, knee, hip, and other joint angles (Markovic et al., 2014). For example, a moderate alteration of countermovement depth markedly affects both the $\mathrm{F}$ and $\mathrm{P}$ output of maximum $\mathrm{CMJ}$, while the maximum $\mathrm{V}$ as assessed from the jump height remains virtually unchanged (Mandic et al., 2015). This phenomenon generally suggests that the movement pattern adaptation to altered loads could confound the relationship between the recorded F and V (Mandic et al., 2015; Markovic et al., 2014).

The face validity of the F-V relationship parameters has been generally accepted (Jaric, 2015). Specifically, the parameters should depict the capacities of the tested muscles to provide high $\mathrm{F}$ (i.e., through $\left.\mathrm{F}_{0}\right), \mathrm{V}\left(\mathrm{V}_{0}\right)$ and $\mathrm{P}$ output $\left(\mathrm{P}_{0}\right)$ when performing the tested tasks. The same parameters also revealed at least moderate concurrent validity when compared with directly measured muscular F, V and P (Cuk et al., 2014; Garcia-Ramos et al., 2016; Giroux et al., 2015; Sreckovic et al., 2015). Therefore, it is not surprising that $\mathrm{CMJ}$ reveals distinctively higher $\mathrm{F}_{0}$ and $\mathrm{P}_{0}$ than the remaining 3 tests, since it is the only one that involves a simultaneous action of large leg and trunk muscles. Note also that the magnitude of the relationship parameters is closely in line with that previously observed in various types of vertical jumps (Cuk et al., 2014; Feeney et al., 2016; Giroux et al., 2015) and bench press throws (Garcia-Ramos et al., 2016; SanchezMedina et al., 2014; Sreckovic et al., 2015). Markedly higher relative variability of the parameters observed in CMJ could be explained by a prominent adaptation of the movement pattern to the altered external load.

F-V relationships observed in two types of variables have so far only been directly compared in vertical jumps (Cuk et al., 2014) and bench press throws (Sreckovic et al., 2015). The results revealed both the similar magnitude of the relationship parameters and similar differences between them when observed from two types of variables. As expected, $\mathrm{P}_{0}$ was markedly higher when obtained from maximum than from the averaged $\mathrm{F}$ and $\mathrm{V}$ variables. This difference mainly originated from the differences in $V_{0}$, rather than from $\mathrm{F}_{0}$. As a result, F-V relationships were steeper for the averaged than for maximum $\mathrm{F}$ and $\mathrm{V}$ variable types. In general, the results obtained from all 4 tests suggest that the F-V relationship could be obtained from both types of $\mathrm{F}$ and $\mathrm{V}$ variables, but the expected differences in the magnitude of their parameters should be strictly kept in mind. Regarding future routine testing procedures, we could recommend the selection of maximum $\mathrm{F}$ and $\mathrm{V}$ values, since they do not require the assessment of the instants of movement initiation and termination as the averaged $\mathrm{F}$ and $\mathrm{V}$ values do.

A frequent implicit presumption of routine tests of physical capacities is that the outcomes typically observed in very few tests could be partly generalized to the entire muscular system. Regarding the present study, the question is whether the individual muscle capacities to produce high $\mathrm{F}, \mathrm{V}$ and $\mathrm{P}$ output assessed by the F$\mathrm{V}$ relationship parameters $\mathrm{F}_{0}, \mathrm{~V}_{0}$ and $\mathrm{P}_{0}$, respectively, can be generalized across all 4 applied tests. The results were inconsistent revealing on average only moderate correlation coefficients among the same parameters obtained from different tests. The stronger relationships observed among $P_{0}$ then among $\mathrm{V}_{0}$ values could be explained by the fact that the tests were conducted in the vicinity of $P_{0}$ (i.e., the region of 
intermediate values of $\mathrm{F}$ and $\mathrm{V}$ ), while $\mathrm{V}_{0}$ mainly represented an outcome of distant extrapolation. Nevertheless, note that the observed moderate generalizability of the F-V parameters could be in line with recent studies revealing weak generalizability of strength measures across the individual muscles of the muscular system (Bohannon, 2008) or functional movements (Pojednic et al., 2012). Note that different variables observed by employing different methods might strongly correlate within the same muscle, but weakly across the muscular system in general (Bozic et al., 2013; Prebeg et al., 2013).

Of particular importance could be practical implications of the obtained findings. When compared with standard tests typically conducted on a single muscle group and under a single mechanical condition, various loaded functional movement tests could selectively reveal the muscle capacities for providing high $\mathrm{F}, \mathrm{V}$ and $\mathrm{P}$ output through the obtained linear F-V relationship. Such a set of information could be highly valuable both in non-clinical (physical education, sports, ergonomy) and clinical areas (physical medicine, rehabilitation, physical therapy). Note also that the functional movements also provide high ecological validity and reliability, as well as at least moderate concurrent validity of the relationship parameters. Finally, a recently proposed 'two-load method' (Jaric, 2016) suggests that the same muscle capacities could be assessed by applying just 2 distinctive loads. All those findings collectively speak in favor of routine testing of muscle capacities through functional movements conducted under several, if not only 2 distinctive mechanical conditions.

To conclude, the present study conducted on both a variety of maximum performance tests and different types of $\mathrm{F}$ and $\mathrm{V}$ variables revealed a rather consistent set of data regarding the modeled F-V relationships. Namely, the relationship proved to be exceptionally strong and linear independently of the test and variable type. Nevertheless, due to their relative simplicity, one could recommend using maximum values of $\mathrm{F}$ and $\mathrm{V}$, rather than their averaged values. Although their generalizability could be limited across different tasks, the obtained relationship parameters discern among important mechanical capacities of the tested muscles. Taking into account both the comprehensive information regarding the muscle mechanical capacities and promising possibilities of its application in routine testing, this line of research certainly deserves attention of both investigators and practitioners.

\section{Acknowledgements}

The study was supported by NIH under grant R21AR06065, and by the Serbian Research Council under grant 175037.

\section{References}

Allison SJ, Brooke-Wavell K, Folland JP. Multiple joint muscle function with ageing: the force-velocity and power-velocity relationships in young and older men. Aging Clin Exp Res, 2013; 25: 159-66

Bohannon RW. Hand-grip dynamometry predicts future outcomes in aging adults. J Geriatr Phys Ther, 2008; 31: 3-10

Bozic PR, Celik O, Uygur M, Knight CA, Jaric S. Evaluation of novel tests of neuromuscular function based on brief muscle actions. J Strength Cond Res, 2013; 27: 1568-78

Cohen J. Statistical power analysis for the behavioral sciences. Statistical Power Analysis for the Behavioral Sciences, 567; 1988

Cuk I, Markovic M, Nedeljkovic A, Ugarkovic D, Kukolj M, Jaric S. Force-velocity relationship of leg extensors obtained from loaded and unloaded vertical jumps. Eur J Appl Physiol, 2014; 114: 1703-14

Feeney D, Stanhope SJ, Kaminski TW, Machi A, Jaric S. Loaded vertical jumping: force-velocity relationship, work, and power. J Appl Biomech, 2016; 32: 120-7 
Garcia-Ramos A, Jaric S, Padial P, Feriche B. Force-velocity relationship of upper body muscles: traditional versus ballistic bench press. J Appl Biomech, 2016; 32: 178-85

Giroux C, Rabita G, Chollet D, Guilhem G. What is the best method for assessing lower limb force-velocity relationship? Int J Sports Med, 2015; 36: 143-9

Hahn D, Herzog W, Schwirtz A. Interdependence of torque, joint angle, angular velocity and muscle action during human multi-joint leg extension. Eur J Appl Physiol, 2014; 114: 1691-702

Jaric S. Force-velocity relationship of muscles performing multi-joint maximum performance tasks. Int J Sports Med, 2015; 36: 699-704

Jaric S. Two-loads method for distinguishing among the muscle force, velocity, and power producing capacities. Sports Med, 2016; 46: 1585-9

Jaskolska A, Goossens P, Veenstra B, Jaskolski A, Skinner JS. Comparison of treadmill and cycle ergometer measurements of force-velocity relationships and power output. Int J Sports Med, 1999; 20: 192-7

Limonta E, Sacchi M. Morphological analysis of force/velocity relationship in dynamic exercise at varying loads. J Strength Cond Res, 2010; 24: 2065-72

Mandic R, Jakovljevic S, Jaric S. Effects of countermovement depth on kinematic and kinetic patterns of maximum vertical jumps. J Electromyogr Kinesiol, 2015; 25: 265-72

Markovic S, Mirkov DM, Nedeljkovic A, Jaric S. Body size and countermovement depth confound relationship between muscle power output and jumping performance. Hum Mov Sci, 2014; 33: 203-10

Pojednic RM, Clark DJ, Patten C, Reid K, Phillips EM, Fielding RA. The specific contributions of force and velocity to muscle power in older adults. Exp Gerontol, 2012; 47: 608-13

Prebeg G, Cuk I, Suzovic D, Stojiljkovic S, Mitic D, Jaric S. Relationships among the muscle strength properties as assessed through various tests and variables. J Electromyogr Kinesiol, 2013; 23: 455-61

Rabita G, Dorel S, Slawinski J, Saez-de-Villarreal E, Couturier A, Samozino P, Morin JB. Sprint mechanics in world-class athletes: a new insight into the limits of human locomotion. Scand J Med Sci Sports, 2015; 25: 583-94

Samozino P, Rejc E, Di Prampero PE, Belli A, Morin JB. Optimal force-velocity profile in ballistic movements--altius: citius or fortius? Med Sci Sports Exerc, 2012; 44: 313-22

Samozino P, Rejc E, di Prampero PE, Belli A, Morin JB. Force-Velocity Properties' Contribution to Bilateral Deficit during Ballistic Push-off. Med Sci Sports Exerc, 2014; 46: 107-14

Sanchez-Medina L, Gonzalez-Badillo JJ, Perez CE, Pallares JG. Velocity- and power-load relationships of the bench pull vs. bench press exercises. Int J Sports Med, 2014; 35: 209-16

Sreckovic S, Cuk I, Djuric S, Nedeljkovic A, Mirkov D, Jaric S. Evaluation of force-velocity and powervelocity relationship of arm muscles. Eur J Appl Physiol, 2015; 115: 1779-87

Taylor-Piliae RE, Norton LC, Haskell WL, Mahbouda MH, Fair JM, Iribarren C, Go AS, Fortmann SP. Validation of a new brief physical activity survey among men and women aged 60-69 years. Am J Epidemiol, 2006; 164: 598-606

Yamauchi J, Mishima C, Nakayama S, Ishii N. Force-velocity, force-power relationships of bilateral and unilateral leg multi-joint movements in young and elderly women. J Biomech, 2009; 42: 2151-7

\section{Corresponding author:}

\section{Dr. Slobodan Jaric}

Rust Arena, Rm. 143

University of Delaware

541 South College Avenue, Newark, DE 19716

Phone: +1-302-8316174

Fax: +1-302-8313693

E-Mail: jaric@udel.edu 